\title{
Estimating complete band diagrams of non-ideal heterointerfaces by combining ellipsometry and photoemission spectroscopy
}

\author{
Crovetto, Andrea
}

Published in:

Journal of Applied Physics

Link to article, DOI:

$10.1063 / 1.5034774$

Publication date:

2018

Document Version

Publisher's PDF, also known as Version of record

Link back to DTU Orbit

Citation (APA):

Crovetto, A. (2018). Estimating complete band diagrams of non-ideal heterointerfaces by combining ellipsometry and photoemission spectroscopy. Journal of Applied Physics, 124(8). https://doi.org/10.1063/1.5034774

\section{General rights}

Copyright and moral rights for the publications made accessible in the public portal are retained by the authors and/or other copyright owners and it is a condition of accessing publications that users recognise and abide by the legal requirements associated with these rights.

- Users may download and print one copy of any publication from the public portal for the purpose of private study or research.

- You may not further distribute the material or use it for any profit-making activity or commercial gain

- You may freely distribute the URL identifying the publication in the public portal

If you believe that this document breaches copyright please contact us providing details, and we will remove access to the work immediately and investigate your claim 


\section{Estimating complete band diagrams of non-ideal heterointerfaces by combining ellipsometry and photoemission spectroscopy}

Andrea Crovetto

Citation: Journal of Applied Physics 124, 085302 (2018); doi: 10.1063/1.5034774

View online: https://doi.org/10.1063/1.5034774

View Table of Contents: http://aip.scitation.org/toc/jap/124/8

Published by the American Institute of Physics

\section{Articles you may be interested in}

Requirements for efficient hole extraction in transition metal oxide-based silicon heterojunction solar cells Journal of Applied Physics 124, 085702 (2018); 10.1063/1.5045250

Unusual elasticity of monoclinic $\mathrm{\beta}-\mathrm{Ga}_{2} \mathrm{O}_{3}$

Journal of Applied Physics 124, 085102 (2018); 10.1063/1.5047017

Investigation of the thermal stability of $\mathrm{MoO}_{\mathrm{X}}$ as hole-selective contacts for $\mathrm{Si}$ solar cells

Journal of Applied Physics 124, 073106 (2018); 10.1063/1.5041774

Analysis of pressure diffusion-wave fields in matrix-fracture media using Green functions of frequency domain Journal of Applied Physics 124, 074902 (2018); 10.1063/1.5040667

Effect of peak stress and tensile strain-rate on spall in tantalum Journal of Applied Physics 124, 085901 (2018); 10.1063/1.5045045

Dynamic acousto-elastic response of single fatigue cracks with different microstructural features: An experimental investigation

Journal of Applied Physics 124, 075303 (2018); 10.1063/1.5036531

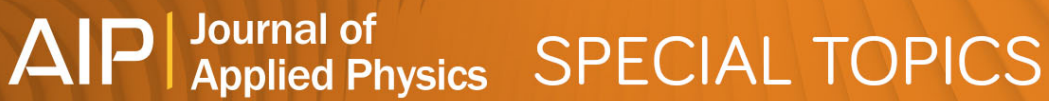

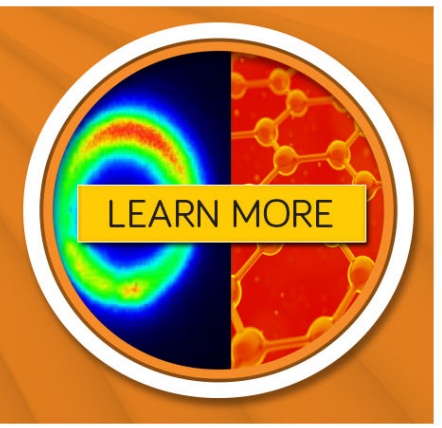




\title{
Estimating complete band diagrams of non-ideal heterointerfaces by combining ellipsometry and photoemission spectroscopy
}

\author{
Andrea Crovetto ${ }^{1,2, a)}$ \\ ${ }^{1}$ DTU Nanotech, Technical University of Denmark, DK-2800 Kgs. Lyngby, Denmark \\ ${ }^{2}$ SurfCat, Department of Physics, Technical University of Denmark, DK-2800 Kgs. Lyngby, Denmark
}

(Received 12 April 2018; accepted 3 August 2018; published online 22 August 2018)

\begin{abstract}
In this work, we show that spectroscopic ellipsometry can be combined with photoemission spectroscopy to obtain complete interface band diagrams of non-ideal semiconductor heterointerfaces, such as interfaces between thin-film polycrystalline materials. The non-destructive ellipsometry measurement probes the near-interface bandgap of the two semiconductors (including the buried semiconductor) after the interface has formed. This is important in the non-ideal case where chemical processes during interface growth modify the electronic properties of the two separated surfaces. Knowledge of near-interface bandgaps improves accuracy in conduction band offset measurements of non-ideal interfaces, and it sheds light on their device physics. Both of those positive outcomes are demonstrated in the $\mathrm{Cu}_{2} \mathrm{ZnSnS}_{4} / \mathrm{CdS}$ interface used here as a case study, where the bandgap of both materials decreases by up to $200 \mathrm{meV}$ from the bulk to the near-interface region. This finding reveals a preferential electron-hole recombination channel near the interface, and it yields corrected values for the interfacial conduction band offset. Published by AIP Publishing.

https://doi.org/10.1063/1.5034774
\end{abstract}

\section{INTRODUCTION}

Conduction band offset measurements of semiconductor heterointerfaces are typically based on a set of direct photoemission spectroscopy measurements. ${ }^{1}$ Those measurements are sensitive to the energy position of the valence band maximum at the semiconductor surface, that is, within a depth of $1-20 \mathrm{~nm}$ depending on the excitation wavelength (UV light or X-rays). To access the conduction band positions, two possibilities exist. The first is simply to measure the bulk bandgap of each semiconductor by an optical technique, such as UV-visible transmission/reflection spectroscopy. ${ }^{2}$ The second is to perform two complementary inverse photoemission spectroscopy measurements (IPES), which yield the energy position of the conduction band minima of the two semiconductor surfaces with an analysis depth of a few $\mathrm{nm} .^{3}$ In the first (second) case, the bulk (surface) bandgaps of the two semiconductors are added to the valence band offset to determine the conduction band offset.

However, formation of a polycrystalline semiconductor heterointerface often results in considerable interdiffusion of chemical species across the interface. Various authors have reported significant interdiffusion at interfaces of interest for thin-film heterojunction solar cells, such as $\mathrm{CdTe} / \mathrm{CdS},{ }^{4}$ $\mathrm{Cu}(\mathrm{In}, \mathrm{Ga}) \mathrm{Se}_{2} / \mathrm{CdS},{ }^{5} \mathrm{Cu}_{2} \mathrm{ZnSn}(\mathrm{S}, \mathrm{Se})_{4} / \mathrm{CdS},{ }^{6}$ and $\mathrm{Cu}_{2} \mathrm{O} / \mathrm{ZnO}{ }^{7}$ Interdiffusion may be enhanced by heating the interface during or after growth, by energetic particle bombardment during growth, by the presence of grain boundaries as a preferential diffusion channel, and by the presence of voids and extended defects in the polycrystalline films. Due to the diffusion and lattice incorporation of elements from the foreign semiconductor, the band structure of each

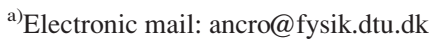

semiconductor in the near-interface region may be significantly different from the respective bulk and near-surface band structure.

In this paper, we apply spectroscopic ellipsometry to non-destructively measure the near-interface bandgaps of two heterojunction semiconductors after interface formation, including the near-interface bandgap of the buried semiconductor. Conduction band offsets can then be derived by adding the near-interface bandgaps to the valence band offset determined with a standard photoemission-based method. Besides improving the accuracy of a conduction band offset measurement, knowledge of the interface bandgaps of a semiconductor heterojunction can help understand its device physics. In fact, both bandgap widening ${ }^{8}$ and bandgap narrowing 9 at the interface can have major consequences at the device level.

\section{SAMPLE PREPARATION}

$\mathrm{Cu}_{2} \mathrm{ZnSnS}_{4} / \mathrm{CdS}(\mathrm{CZTS} / \mathrm{CdS})$ interfaces processed at different temperatures were employed as a case study for the proposed experimental method. The reason for choosing this particular interface is the ease of $\mathrm{Cd}$ diffusion from $\mathrm{CdS}$ to CZTS, as reported by many authors. ${ }^{10}$ The resulting $\mathrm{Cu}_{2}(\mathrm{Zn}, \mathrm{Cd}) \mathrm{SnS}_{4}$ solid solution present in the region of interdiffusion is expected to have a lower bandgap than pure CZTS, ${ }^{11}$ so the CZTS/CdS interface is an ideal system to test the proposed experimental method for detecting nearinterface bandgap changes. CZTS thin films (about $100 \mathrm{~nm}$ ) were deposited on a soda lime glass substrate by pulsed laser deposition from a single target with CZTS stoichiometry. Careful control of the laser fluence enabled deposition of nearly amorphous CZTS precursors with the desired stoichiometry, ${ }^{12}$ which were further sulfurized at $550{ }^{\circ} \mathrm{C}$ for $10 \mathrm{~min}$. Process details and film characterization are 
available elsewhere. ${ }^{12}$ Three CdS thin films (20-40 nm) were then grown on nominally identical glass/CZTS substrates by chemical bath deposition at $55^{\circ} \mathrm{C}, 75^{\circ} \mathrm{C}$, and $95^{\circ} \mathrm{C}$. Chemical bath deposition is the standard technique used in the highest efficiency CZTS solar cells. ${ }^{13,14}$ After $\mathrm{CdS}$ deposition, each of the three samples was cut into two halves, and one half of each sample was post-annealed for 20 minutes at $300{ }^{\circ} \mathrm{C}$ in an $\mathrm{Ar}$ atmosphere. A total of six samples were therefore available for the present study. As will be shown in Sec. III, simultaneous treatment of the data measured on several, slightly different samples (multi-sample analysis) can considerably improve the reliability of ellipsometry results.

\section{MEASUREMENT OF NEAR-INTERFACE BANDGAPS}

\section{A. Theory}

In an ellipsometry measurement in reflection mode with incidence angle $\theta$, the polarization state of the incident beam can be described by the amplitude and phase of the two polarization components $s$ (perpendicular to the plane of incidence) and $p$ (contained in the plane of incidence). One can then define $r_{\mathrm{s}}(E)$ and $r_{\mathrm{p}}(E)$ as the complex reflection coefficients of the sample as a function of photon energy for the $s$ and $p$ polarization components, respectively. By measuring the polarization state of the reflected beam and comparing it to the known polarization state of the incident beam, the ellipsometer determines the complex ratio $r_{\mathrm{p}}(E) / r_{\mathrm{s}}(E)$ of the two reflection coefficients. That ratio is typically expressed by the two real numbers $\Psi(E)$ and $\Delta(E)$, where

$$
\frac{r_{\mathrm{p}}(E)}{r_{\mathrm{s}}(E)}=\tan [\Psi(E)] e^{i \Delta(E)} .
$$

For a sample consisting of a number of stacked flat layers, each with a known thickness $d$ and dielectric function $\varepsilon(E)$, the overall reflection coefficients $r_{\mathrm{p}}(E)$ and $r_{\mathrm{s}}(E)$ at a given incidence angle $\theta$ can be determined by theory using the Fresnel equations to obtain the reflection coefficient of each interface and a correction factor to account for interference effects due to the finite thickness of each layer [Fig. 1(a)]. Therefore, $\Psi(E)$ and $\Delta(E)$ spectra can be completely determined by theory as a function of the thicknesses and dielectric functions of all layers. If the problem is inverted, that is, if some of the thicknesses and dielectric functions involved are unknown, they can in principle be determined by regression analysis through least-squares fitting of the measured $\Psi(E)$ and $\Delta(E)$ spectra using the unknown quantities as fitting parameters. This is the principle of spectroscopic ellipsometry. ${ }^{15}$

The goal of this work is to determine the bandgaps of CZTS and CdS near the CZTS/CdS interface, where they are potentially modified by chemical processes with respect to the bulk. Based on the discussion above, this can be done by spectroscopic ellipsometry with the following procedure: (i) acquiring $\Psi(E)$ and $\Delta(E)$ spectra from a glass/ CZTS/CdS sample; (ii) modeling the CZTS and CdS layers as several sub-layers, each of which has an independent dielectric function [thus an independent bandgap, Fig.

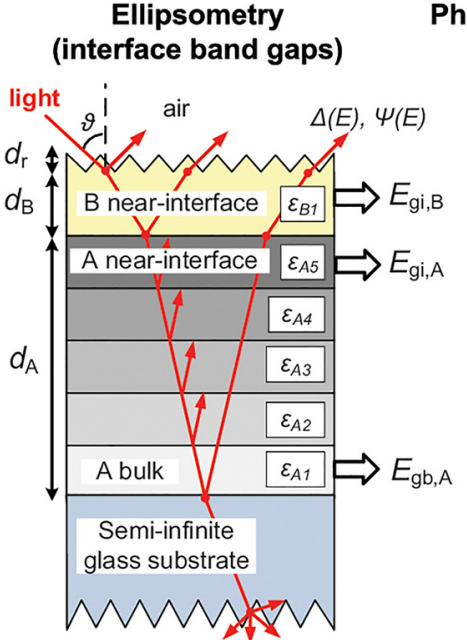

(a)
Photoemission spectroscopy (valence band offsets)

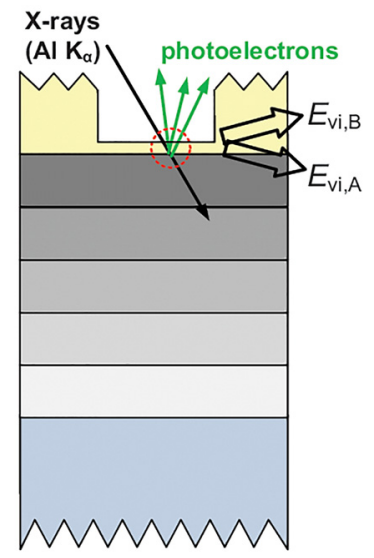

(b)
FIG. 1. (a): Scheme of the optical model used to extract the bandgaps of CZTS ("A") and CdS ("B") by fitting the ellipsometry spectra $\Psi(E)$ and $\Delta(E)$. The bandgaps $E_{\mathrm{gb}, \mathrm{A}}, E_{\mathrm{gi}, \mathrm{A}}$, and $E_{\mathrm{gi}, \mathrm{B}}$ are derived from the fitted dielectric functions $\varepsilon_{\mathrm{A} 1}, \varepsilon_{\mathrm{A} 5}$, and $\varepsilon_{\mathrm{B} 1}$ of the corresponding layers. (b): Scheme of the XPS measurement employed to determine the valence band offset $\mathrm{VBO}=E_{\mathrm{vi}, \mathrm{B}}-E_{\mathrm{vi}, \mathrm{A}}$. Refer to the main text for the meaning of the symbols.

1(a)]; (iii) parameterizing the dielectric function of each layer with a number of fitting parameters which, together with the unknown layer thicknesses, are used to fit the measured $\Psi(E)$ and $\Delta(E)$ spectra (Fig. 2); and (iv) extracting the bandgap value of each layer based on the shape of the fitted imaginary part of the dielectric function near the absorption onset [Fig. 3(a)].

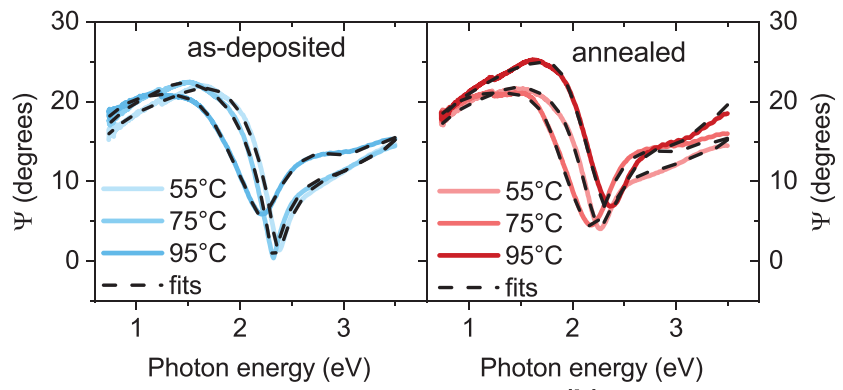

(a)

(b)

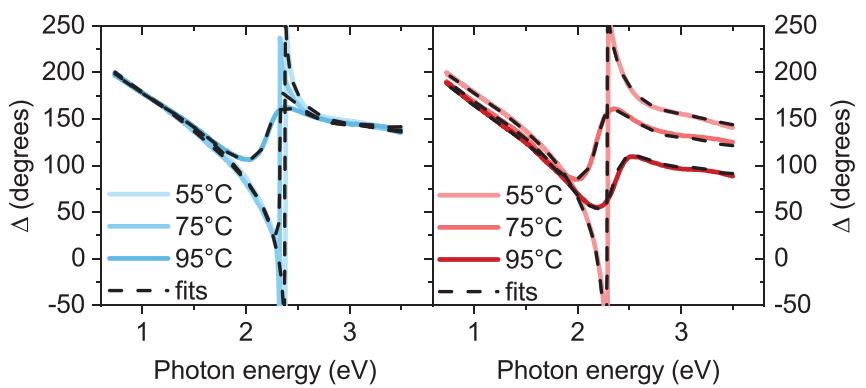

(c)

(d)

FIG. 2. $\Psi(E)$ spectra [(a) and (b)] and $\Delta(E)$ spectra [(c) and (d)] of the six investigated samples. Three samples are measured as-deposited [(a) and (c)] and three are measured after post-annealing [(b) and (d)]. For readability, only the spectra taken at an angle of incidence of $60^{\circ}$ are included. The measured and fitted spectra at all (six) incidence angles are shown in the supplementary material. 


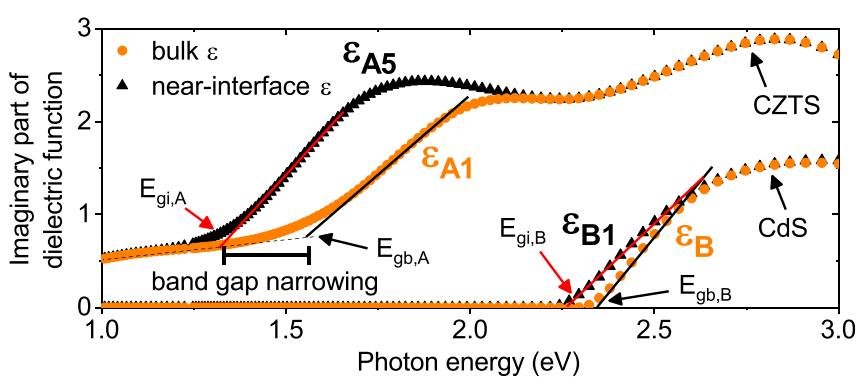

(a)

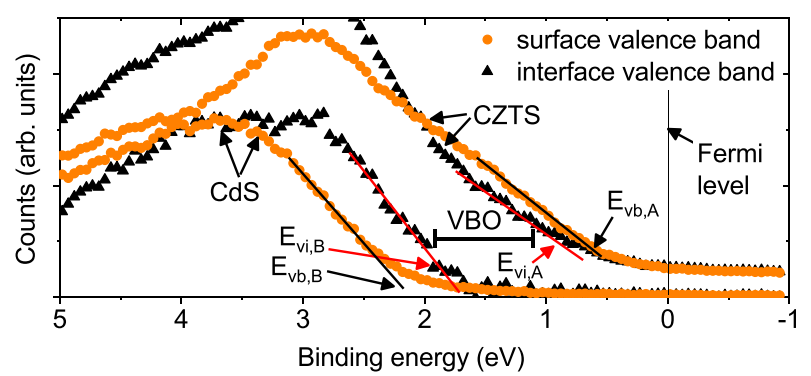

(b)

FIG. 3. (a): The fitted imaginary part of the dielectric function of various layers of one sample in this study (CdS deposition temperature of $55^{\circ} \mathrm{C}$, with post-annealing). Note that $\varepsilon_{\mathrm{B}}$ is obtained from a separate ellipsometry measurement of a CdS film grown on ITO-coated glass. The corresponding bandgaps are extracted by extrapolating the absorption onset with a straight line. Refer to Fig. 1(a) and to the main text for interpreting the symbols. (b) Relevant photoemission onsets for the same sample as above. Valence band positions are obtained by extrapolating photoemission onsets with a straight line. Note that $E_{\mathrm{vi}, \mathrm{B}}$ is derived from the same photoemission spectrum as $E_{\mathrm{vi}, \mathrm{A}}$ by the valence band difference method. ${ }^{26}$ Note also that the two baresurface valence band spectra are measured on an in-situ-cleaned bare CZTS surface and on an in-situ-cleaned bare CdS surface on an ITO-coated glass substrate. Refer Fig. 1(b) and the main text for interpreting the symbols.

\section{B. Experimental details and analysis method}

$\Psi(E)$ and $\Delta(E)$ spectra were acquired in the reflection mode with a rotating compensator ellipsometer (M-2000, J.A. Woollam Co.). The spot size was about $200 \mu \mathrm{m} \times 300 \mu \mathrm{m}$, and the photon energy range was $0.78 \mathrm{eV} \leq E \leq 3.50 \mathrm{eV}$. The measurement was repeated at six different incidence angles $\theta$ for each sample $\left(45^{\circ} \leq \theta \leq 70^{\circ}\right)$ to provide a large dataset for more robust fitting. The CompleteEase software package (version 5.06, J.A. Woollam Co.) was used for fitting work.

The layer model employed in this study is shown in Fig. 1(a). One important remark is that, while the expected $\Psi(E)$ and $\Delta(E)$ of a known sample are unique and can be calculated analytically, the uniqueness of the solution to the inverse problem (i.e., fitting the measured $\Psi(E)$ and $\Delta(E)$ to determine unknown properties of the samples) is not guaranteed. Hence, the sample should be modeled with the smallest possible number of fitting parameters, and the largest possible number of independent spectra should be fitted simultaneously against a consistent model. For this reason, the 36 $\Psi(E)$ spectra and the $36 \Delta(E)$ spectra measured in this work (6 samples times 6 angles of incidence) were all fitted simultaneously, as detailed later in the article as well as in the supplementary material.

The CZTS layer ("A") has unknown thickness $d_{\mathrm{A}}$ (fitting parameter) and unknown, possibly depth-dependent dielectric function due to interdiffusion. To model a depthdependent dielectric function at the smallest possible expense of fitting parameters, we slice the CZTS layer into five separate sub-layers of equal thickness [Fig. 1(a)]. The dielectric function of the bottom layer $\varepsilon_{\mathrm{A} 1}(E)$ is parameterized with a Kramers-Kronig-consistent b-spline function with 3 nodes/eV, corresponding to 10 fitting parameters in the measured range $0.78 \mathrm{eV} \leq E \leq 3.50 \mathrm{eV}$, similar to previous ellipsometry work on CZTS. ${ }^{16,17}$ The possible bandgap change from the bottom layer (bulk CZTS) to the top layer (near-interface CZTS) is modeled by allowing the b-spline node located at $1.68 \mathrm{eV}$ to vary linearly from the bottom layer to the top layer. All other nodes are kept constant through the layers. A linear gradient in the b-spline node, corresponding to a linear bandgap gradient across the CZTS layer, is chosen under the assumption that the bandgap changes in CZTS are mostly caused by $\mathrm{Cd}$ interdiffusion and that the Cd content in CZTS decreases steadily with increasing distance from the CdS interface, with a decay length comparable with the CZTS film thickness. Such assumptions are compatible with the existing knowledge of interdiffusion processes at the CZTS/CdS interface, which is summarized in a review paper. ${ }^{10}$ Note that, independently of the number of sub-layers used to model bandgap grading in CZTS, the bspline node at $1.68 \mathrm{eV}$ in the dielectric function $\varepsilon_{\mathrm{A} 5}(E)$ of the top CZTS layer is the only additional fitting parameter with respect to the case of a depth-independent dielectric function. As the $\mathrm{Cd}$ content in CZTS is expected to decay smoothly (instead of abruptly) with depth, the CZTS layer should in principle be modeled with an infinite number of sub-layers. In practice, we noticed that a number of sublayers higher than five did not improve the goodness of the fit further and did not change the best-fit parameters (see the supplementary material).

The dielectric function $\varepsilon_{\mathrm{B} 1}(E)$ of the CdS layer ("B") could also be depth-dependent in principle. However, slicing $\mathrm{CdS}$ into two sub-layers with varying dielectric function only led to marginal improvement of the mean squared error (MSE) of the fit, from 11.0 to 10.8 (supplementary material). With two CdS sublayers, we also observed poor reproducibility of the best-fit parameters upon variation of the initial guess, as well as an increase in the error bars of the $\mathrm{CdS}$ bandgap by an average of $68 \%$. This may indicate that the bandgap of CdS is roughly depth-independent up to a few tens of nm away from the interface with CZTS, or that a depth-dependent dielectric function is difficult to capture for such a thin layer, due to overparameterization. CdS is thus modeled as a single layer with unknown thickness $d_{\mathrm{B}}$ and unknown dielectric function $\varepsilon_{\mathrm{B} 1}(E)$. The imaginary part of $\varepsilon_{\mathrm{B} 1}(E)$ is parameterized with one simplified Tauc-Lorentz oscillator and one pole in the ultraviolet, and the real part of $\varepsilon_{\mathrm{B} 1}(E)$ is derived by the subsequent Kramers-Kronig integration. The parameters of a Tauc-Lorentz oscillator are the amplitude $A$, broadening $B$, and energy $E_{\mathrm{o}}$ of a Lorentzian function, plus a bandgap energy parameter $E_{\mathrm{g}}$ and a highfrequency dielectric function parameter $\varepsilon_{\infty} .{ }^{18}$ Enforcing the conditions $\varepsilon_{\infty}=1$ and $E_{\mathrm{o}}=E_{\mathrm{g}}$ does not have noticeable effects on the MSE of our study. This limits the number of fitted parameters for the Tauc-Lorentz oscillator to 3 . The 
additional node in the ultraviolet has amplitude $A_{\mathrm{uv}}$ and energy $E_{\mathrm{uv}}$ as fitting parameters.

Surface roughness has a strong influence on the $\Psi(E)$ and $\Delta(E)$ spectra and can complicate ellipsometry analysis if it exceeds a few nm. ${ }^{19}$ Roughness was minimized in the sample preparation phase by intentionally growing relatively thin $(100 \mathrm{~nm})$ CZTS layers. In the fitting phase, the roughness layer is modeled by means of Bruggeman's effective medium theory, assuming a 50\%/50\% mix of air and CdS in the roughness layer. ${ }^{15}$ This requires 1 fitting parameter $d_{\mathrm{r}}$ for the thickness of the roughness layer [Fig. 1(a)]. The roughness values determined by ellipsometry were double checked by measuring the root mean square roughness for each sample by atomic force microscopy (AFM), with reasonable agreement between the two techniques (see the supplementary material). The dielectric function of the glass substrate was determined from a separate ellipsometry measurement on bare glass and is kept fixed when fitting CZTS/CdS ellipsometry spectra. The thickness of the glass substrate does not influence the $\Psi(E)$ and $\Delta(E)$ spectra because opaque tape was applied to the back side of the glass to suppress back-side reflection. ${ }^{20}$

To further reduce the chance of overparameterizing the fitting problem, the $\Psi(E)$ and $\Delta(E)$ spectra from the six samples investigated in this work are all fitted simultaneously, using an approach known as the multi-sample analysis. ${ }^{15,21}$ The parameters that are expected to vary significantly from sample to sample are fitted independently in each sample. Those 6 parameters are $d_{\mathrm{A}}, d_{\mathrm{B}}, d_{\mathrm{r}}, E_{\mathrm{o}}$, as well as the nodes of $\varepsilon_{\mathrm{A} 1}(E)$ and $\varepsilon_{\mathrm{A} 5}(E)$ at $1.68 \mathrm{eV}$. Conversely, the parameters that are expected to be roughly constant from sample to sample are forced to have the same best-fit value across samples. Those 13 parameters are $A, B, A_{\mathrm{uv}}, E_{\mathrm{uv}}$, and the 9 remaining nodes of $\varepsilon_{\mathrm{A} 1}(E)$.

\section{Results}

While the multi-sample analysis approach yields a somewhat higher MSE (11.0) compared to separate fitting of each sample, it also results in a dramatic decrease in the error bars of the best-fit parameters, which are defined based on 90\% confidence intervals. As detailed in the supplementary material, the error bars of the layer thicknesses are roughly $\pm 1 \%$; the error bars of the $E_{\mathrm{o}}$ parameter, related to the $\mathrm{CdS}$ bandgap, are roughly $\pm 0.1 \%$; the error bars of the $\varepsilon_{\mathrm{Al}}(1.68)$ node, related to the CZTS bulk bandgap, are roughly $\pm 1 \%$; and the error bars of the $\varepsilon_{\mathrm{A} 1}(1.68)-\varepsilon_{\mathrm{A} 5}(1.68)$ quantity, related to the CZTS near-interface bandgap, are between $\pm 1 \%$ and $\pm 10 \%$. The best-fit values of all fitting parameters are shown in the supplementary material, together with all the retrieved dielectric functions, the cross-correlation coefficients between parameters, and the residuals of the fit. Knowledge of the best-fit values of the dielectric function parameters for the two materials allows derivation of their bandgaps. Band tailing in CZTS absorbers is a well-known issue, which hinders accurate determination of the bandgap by standard Tauc plots. ${ }^{22}$ Band tails are clearly visible in Fig. 3(a) as the imaginary part of the CZTS dielectric function does not go to zero below the expected bandgap energy. The shape of the real and imaginary parts of the CZTS

dielectric functions extracted in this work (shown in the supplementary material) and the intensity of their band tails are in quantitative agreement with previous measurements. ${ }^{23}$ The very large band tails are most likely caused by a combination of sub-bandgap absorption of CZTS due to potential fluctuations, ${ }^{24}$ as well as due to the presence of low bandgap secondary phases, which are difficult to avoid in a quaternary compound with a small single-phase region. ${ }^{22}$ Since secondary phases are often located preferentially at the back of the CZTS films, they may be included in the optical model at the expense of several fitting parameters, ${ }^{25}$ and this may remove some of the CZTS band tails. We simply estimate the bulkand near-interface bandgap of CZTS $\left(E_{\mathrm{gb}, \mathrm{A}}\right.$ and $E_{\mathrm{gi}, \mathrm{A}}$, respectively) by extrapolating the imaginary part of the corresponding dielectric function $\left(\varepsilon_{\mathrm{A} 1}\right.$ and $\varepsilon_{\mathrm{A} 5}$, respectively) with a straight line, as shown in Fig. 3(a). For consistency, the same procedure is applied to determine the bulk bandgap of $\mathrm{CdS}$ $\left(E_{\mathrm{gb}, \mathrm{B}}\right)$ from $\varepsilon_{\mathrm{B}}$, and the near-interface bandgap of $\mathrm{CdS}$ $\left(E_{\mathrm{gi}, \mathrm{B}}\right)$ from $\varepsilon_{\mathrm{B} 1}$. This simple approach has two advantages: (i) it yields bulk bandgaps in good agreement with the CZTS bandgap as determined by the quantum efficiency analysis of a complete solar cell $(1.56 \mathrm{eV}$ versus $1.53 \mathrm{eV}) ;^{12}$ (ii) it removes part of the ambiguity in the extrapolation of the dielectric function near the absorption onset, since the band tail is easily distinguished from the bandgap [Fig. 3(a)]. Note that the quantity $\varepsilon_{\mathrm{B}}$, and therefore the bulk bandgap of CdS, is obtained from a separate ellipsometry measurement of a CdS film grown on ITO-coated glass. A shown in Figs. 4(a) and 4(b), the general trend for both materials is bandgap narrowing in the near-interface region. Specifically, the bandgap

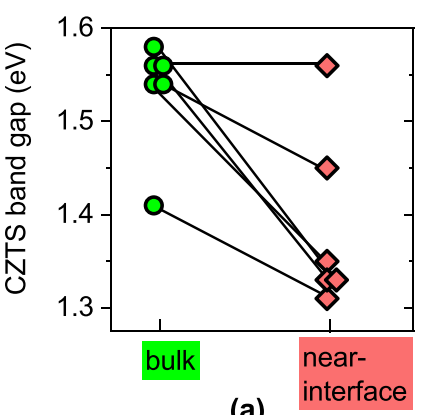

(a)

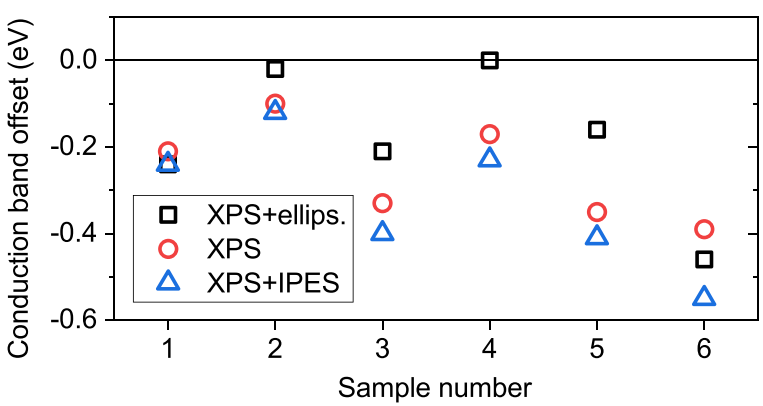

(c)

FIG. 4. (a) The bulk- and near-interface bandgaps of CZTS as determined by ellipsometry for the six samples investigated in this study. The solid lines connect the bulk bandgap $\left(E_{\mathrm{gb}, \mathrm{A}}\right)$ and the near interface bandgap $\left(E_{\mathrm{gi}, \mathrm{A}}\right)$ of the same sample. (b) The bulk- and near-interface bandgaps of CdS as determined by ellipsometry for the same samples. (c) Conduction band offset of each sample calculated with Eq. (2) (squares), with Eq. (3) (circles), and with Eq. (4) (triangles). 
of CZTS decreases by several hundred meV from bulk to the interface, as expected from $\mathrm{Cd}$ interdiffusion. This effect alone can have serious consequences at the device level. ${ }^{9}$

To justify the validity of the optical model employed in this study, we compare it to alternative models. Detailed fitting results of the alternative models are shown in the supplementary material. Here, we focus on the main conclusions. A simpler model would neglect bandgap grading in CZTS and treat CZTS as a homogeneous layer, thus eliminating one fitting parameter $\left(\varepsilon_{\mathrm{A} 5}(1.68)\right)$ per sample. The MSE of this simplified model is 12.1 , compared to 11.0 for the model including bandgap grading. Besides the appreciable MSE improvement, the best-fit results for the model with bandgap grading are in qualitative agreement with the theoretical prediction of near-interface bandgap narrowing in CZTS due to $\mathrm{Cd}$ interdiffusion. The additional fitting parameter results in only a marginal increase $(7 \%)$ of the average error bar for the parameter $\varepsilon_{\mathrm{A} 1}(1.68)$, related to the bulk bandgap of CZTS. An alternative way to model Cd interdiffusion could be to define a new layer between CZTS and CdS using the effective medium approximation (EMA). In such a model, the region of interdiffusion consists of a heterogeneous mix of separated CZTS and CdS phases, rather than a homogeneous $\mathrm{Cu}_{2}(\mathrm{Zn}, \mathrm{Cd}) \mathrm{SnS}_{4}$ solution. The fitting parameter $\varepsilon_{\mathrm{A} 5}(1.68)$ is replaced by the thickness of the EMA layer. Thus, the total number of fitting parameters remains constant. The MSE of the alternative model is noticeably higher (11.8), which indicates that Cd incorporation into CZTS is dominant over heterogeneous mixing of CZTS and CdS. We have also investigated the replacement of linear bandgap grading in CZTS with exponential bandgap grading. Exponential grading should reflect the typical exponential decay of an interdiffusing species more accurately. The problem with this approach is that it requires one additional fitting parameter for the value of the exponent, which is not known a priori. Even though the MSE of the fit (10.7) is somewhat lower than for linear grading, the best-fit exponent takes a negative value, which is the opposite direction than the expected exponential tail of Cd content versus depth in CZTS. Furthermore, unexpected features appear in some of the best-fit dielectric functions of CTZS, which make us opt for the simpler, linearly graded model. Finally, we have tested a model where the region of bandgap grading only covers the top half of the CZTS layer. This would be an appropriate model if the region of interdiffusion was limited to a depth of $30-40 \mathrm{~nm}$. Without changes in the total number of fitted parameters compared to our original model, the MSE of the fit is 11.3 versus 11.0 for the original model. In addition, unexpected features appear in some CZTS dielectric functions similar to the case of exponential grading. We therefore conclude that the optical model employed in this study achieves a reasonable compromise between an oversimplified model and an overparameterized model.

\section{MEASUREMENT OF VALENCE BAND OFFSETS}

To determine the conduction band offsets of the six samples using the near-interface bandgaps measured by ellipsometry, valence band offsets must also be measured. An established experimental technique based on x-ray photoelectron spectroscopy (XPS) was employed, ${ }^{26}$ using a Thermo Scientific K-Alpha instrument with a monochromatized $\mathrm{Al} \mathrm{K} \mathrm{K}_{\alpha}$ X-ray source and a spot size of roughly $400 \mu \mathrm{m}$. The binding energy scale was calibrated with the Fermi level of an in-situ-cleaned Au sample and confirmed by the position of the adventitious $\mathrm{C} 1 \mathrm{~s}$ peak on as-prepared samples. The different CZTS/CdS samples were progressively etched with low-energy $\mathrm{Ar}^{+}$ions $(200 \mathrm{eV})$, and photoemission spectra of the valence band region were recorded after each etching step [Fig. 1(b)]. This ion energy has been shown not to alter the valence band features of CdS and CZTS. ${ }^{2}$ Valence band positions with respect to the Fermi level (zero binding energy) were measured by extrapolating the energy of the photoemission onset of each material with a straight line $e^{3,27}$ as shown in Fig. 3(b). The first spectrum with a recognizable photoemission onset of CZTS was used to determine the interface valence band position of CZTS with respect to the Fermi level $\left(E_{\mathrm{vi}, \mathrm{A}}\right)$. The interface valence band position of $\mathrm{CdS}\left(E_{\mathrm{vi}, \mathrm{B}}\right)$ was derived from the same spectrum by the technique of valence band difference spectra ${ }^{26}$ or "direct VBO method." 10 The interface valence band offset (VBO) was simply calculated as $\mathrm{VBO}=E_{\mathrm{vi}, \mathrm{B}}-E_{\mathrm{vi}, \mathrm{A}}[$ Fig. 3(b)]. The valence band difference method has been applied to CZTS/ $\mathrm{CdS}$ interfaces before, ${ }^{2}$ and it involves deconvoluting the CZTS valence band signal from the total photoemission signal in order to determine the energy of the superimposed $\mathrm{CdS}$ photoemission onset. ${ }^{26}$ The valence band positions of a bare CZTS surface $\left(E_{\mathrm{vb}, \mathrm{A}}\right)$ and of a bare CdS surface $\left(E_{\mathrm{vb}, \mathrm{B}}\right)$ were also measured for reference and are shown in Fig. 3(b). They were measured by XPS on a bare CZTS film on glass and on a CdS film deposited on ITO-coated glass, respectively, after removal of the native oxide layer by $\mathrm{Ar}^{+}$etching at $200 \mathrm{eV}$.

\section{DISCUSSION AND COMPARISON WITH OTHER METHODS}

The combination of ellipsometry- and XPS measurements makes it possible to determine conduction band offsets from valence band offsets using the relevant bandgaps for the problem, i.e., the near-interface bandgaps. As shown in Fig. 4(c), the measured conduction band offsets (CBO) across the six samples span over a $0.4 \mathrm{eV}$ range, partially due to differences in their near-interface bandgaps. It can be interesting to compare the $\mathrm{CBO}$ values determined by the present method to the values that would be obtained by more established methods. For the present method (XPS + ellipsometry)

$$
\mathrm{CBO}=\mathrm{VBO}+E_{\mathrm{gi}, \mathrm{B}}-E_{\mathrm{gi}, \mathrm{A}} .
$$

For a method based on the XPS-determined valence band offset and bulk bandgaps

$$
\mathrm{CBO}=\mathrm{VBO}+E_{\mathrm{gb}, \mathrm{B}}-E_{\mathrm{gb}, \mathrm{A}} .
$$

Even though no IPES measurements were performed in this study, we can assume based on the previous work ${ }^{3}$ that the combination of IPES and XPS would yield a CZTS surface 


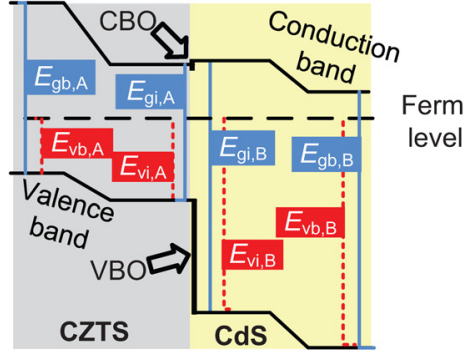

(a)

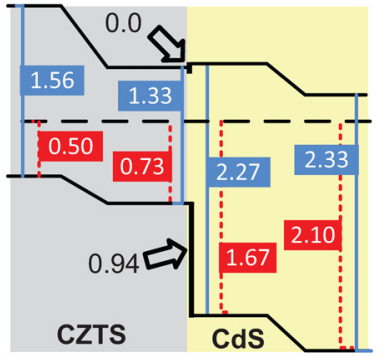

(b)
FIG. 5. (a) Key for relating the quantities measured in this study to interface band diagrams. (b) The specific interface band diagram extracted for the sample with a $\mathrm{CdS}$ deposition temperature of $55^{\circ}$ and post-annealing.

bandgap close to $E_{\mathrm{gb}, \mathrm{A}}$ and a CdS surface bandgap close to $E_{\mathrm{gi}, \mathrm{B}}$ when measuring on a thin $\mathrm{CdS}$ layer deposited on CZTS. Therefore, for a method based on the XPSdetermined valence band offset and the IPES-determined surface bandgaps, we estimate

$$
\mathrm{CBO}=\mathrm{VBO}+E_{\mathrm{gi}, \mathrm{B}}-E_{\mathrm{gb}, \mathrm{A}} .
$$

Figure 4(c) compares the CBOs determined by Eqs. (2)-(4). The underestimation of the CBO by the XPS + IPES method [Eq. (4)] can be explained by the fact that the XPS + IPES method captures near-interface bandgap narrowing in CdS, but not in CZTS. ${ }^{3}$ The less severe CBO underestimation by the XPS method with separately determined bulk bandgaps [Eq. (3)] can be explained by error cancellation, since bandgap changes of both CZTS and CdS near the interface are in the same direction (i.e., towards bandgap narrowing).

Finally, detailed interface band diagrams can be drawn based on the quantities measured in this work, as illustrated in Fig. 5(a). Taking the interface band diagram of the sample with a $\mathrm{CdS}$ deposition temperature of $55^{\circ} \mathrm{C}$ and postannealing as an example [Fig. 5(b)], interesting features that influence the physics of the resulting device are found. The flat conduction band offset is a beneficial feature for electron transport across the interface and minimization of interface recombination. ${ }^{10}$ The downwards band bending from CZTS to $\mathrm{CdS}$ is also a beneficial feature, as it drives electrons and holes towards their respective contacts. Bandgap narrowing in CZTS is, however, a detrimental feature, as it decreases the barrier to recombination in the near-interface region, thus potentially decreasing the maximum achievable solar cell open-circuit voltage. ${ }^{9}$ Understanding the specific chemical mechanisms responsible for bandgap narrowing and their dependence on process conditions is not straightforward and will thus require a separate study. ${ }^{29}$ Here we simply note that near-interface bandgap narrowing of CZTS and CdS is observed consistently in all samples, regardless of their different processing conditions.

\section{METHOD LIMITATIONS}

Our proposed experimental method certainly has a number of limitations. First of all, any effect that modifies the bandgaps of the materials only in a very shallow region of a few $\mathrm{nm}$ across the interface is very difficult to detect by ellipsometry, since the effect of dielectric function changes on $\Psi(E)$ and $\Delta(E)$ spectra for very thin layers can be indistinguishable from thickness changes. ${ }^{28}$ Hence, the present method is only sensitive to "near-interface" bandgap changes (tens of $\mathrm{nm}$ ), which can be related, e.g., to relatively deep interdiffusion or to phase segregation after post-annealing. This is the reason for the expression "near-interface bandgap" used throughout this article. Since ellipsometry is a model-based indirect technique, the quality of the ellipsometry results strongly depends on the correctness of the layer model and on careful selection of fitting parameters, which is a trade-off between oversimplified and overparameterized models. ${ }^{15}$ Finally, the sample preparation phase is critical. Polycrystalline materials with relatively large, micron-sized grains can result in unacceptably high surface roughness, which renders ellipsometry analysis impossible. ${ }^{15}$ This issue can be circumvented by depositing thin layers of materials or by polishing the layers after deposition.

\section{CONCLUSION}

We have proposed an extension to a standard photoemission-based method for obtaining detailed interface band diagrams of semiconductor heterojunctions where chemical processes during interface growth or postannealing modify the interface electronic properties with respect to the bulk or to the bare surface. For the particular heterointerface selected to demonstrate the method $\left(\mathrm{Cu}_{2} \mathrm{ZnSnS}_{4} / \mathrm{CdS}\right)$, a bandgap decrease in several hundred $\mathrm{meV}$ from bulk to interface was observed on both sides of the interface. The resulting device physics is significantly influenced by the bandgap narrowing phenomenon. Adding the near-interface bandgaps to the valence band offsets measured by photoemission spectroscopy significantly improves the accuracy of a standard conduction band offset measurement.

\section{SUPPLEMENTARY MATERIAL}

See supplementary material for more details about possible optical models used to fit ellipsometry spectra, the bestfit values of all fitting parameters, plots of the experimental versus modeled ellipsometry spectra of all the investigated samples, a table with cross-correlation coefficients between fitted parameters, plots of the retrieved dielectric functions, and comparison between the roughness values extracted by ellipsometry and by atomic force microscopy.

\section{ACKNOWLEDGMENTS}

This work was supported by the Danish Council for Strategic Research, the Innovation Fund Denmark (File No. 5016-00102), and VILLUM Fonden (Grant No. 9455). We are grateful to Rebecca Ettlinger and Andrea Cazzaniga for assistance with sample preparation, as well as to Ole Hansen and Jørgen Schou for useful discussions.

\footnotetext{
${ }^{1}$ A. Klein, J. Phys.: Condens. Matter 27, 134201 (2015).

${ }^{2}$ A. Santoni, F. Biccari, C. Malerba, M. Valentini, R. Chierchia, and A. Mittiga, J. Phys. D: Appl. Phys. 46, 175101 (2013).
} 
${ }^{3}$ M. Bär, B.-A. Schubert, B. Marsen, R. G. Wilks, S. Pookpanratana, M. Blum, S. Krause, T. Unold, W. Yang, L. Weinhardt, C. Heske, and H.-W. Schock, Appl. Phys. Lett. 99, 222105 (2011).

${ }^{4}$ J. P. Enríquez, E. Gómez Barojas, R. Silva González, and U. Pal, Sol. Energy Mater. Sol. Cells 91, 1392 (2007).

${ }^{5}$ C. Heske, D. Eich, R. Fink, E. Umbach, T. van Buuren, C. Bostedt, L. J. Terminello, S. Kakar, M. M. Grush, T. A. Callcott, F. J. Himpsel, D. L. Ederer, R. C. C. Perera, W. Riedl, and F. Karg, Appl. Phys. Lett. 74, 1451 (1999).

${ }^{6}$ M. Bär, I. Repins, L. Weinhardt, J.-H. Alsmeier, S. Pookpanratana, M. Blum, W. Yang, C. Heske, R. G. Wilks, and R. Noufi, ACS Energy Lett. 2, 1632 (2017).

${ }^{7}$ S. S. Wilson, J. P. Bosco, Y. Tolstova, D. O. Scanlon, G. W. Watson, and H. A. Atwater, Energy Environ. Sci. 7, 3606 (2014).

${ }^{8}$ M. Bär, S. Nishiwaki, L. Weinhardt, S. Pookpanratana, O. Fuchs, M. Blum, W. Yang, J. D. Denlinger, W. N. Shafarman, and C. Heske, Appl. Phys. Lett. 93, 244103 (2008).

${ }^{9}$ A. Crovetto, M. Palsgaard, T. Gunst, T. Markussen, K. Stokbro, M. Brandbyge, and O. Hansen, Appl. Phys. Lett. 110, 083903 (2017); e-print arXiv: 1702.04229 .

${ }^{10}$ A. Crovetto and O. Hansen, Sol. Energy Mater. Sol. Cells 169, 177 (2017).

${ }^{11}$ Z. Su, J. M. R. Tan, X. Li, X. Zeng, S. K. Batabyal, and L. H. Wong, Adv. Energy Mater. 5, 1500682 (2015).

${ }^{12}$ A. Cazzaniga, A. Crovetto, C. Yan, K. Sun, X. Hao, J. Ramis Estelrich, S. Canulescu, E. Stamate, N. Pryds, O. Hansen, and J. Schou, Sol. Energy Mater. Sol. Cells 166, 91 (2017).

${ }^{13}$ S. Tajima, M. Umehara, M. Hasegawa, T. Mise, and T. Itoh, Prog. Photovoltaics: Res. Appl. 25, 14 (2017).

${ }^{14}$ F. Liu, J. Huang, K. Sun, C. Yan, Y. Shen, J. Park, A. Pu, F. Zhou, X. Liu, J. A. Stride, M. A. Green, and X. Hao, NPG Asia Mater. 9, e401 (2017).
${ }^{15}$ H. Fujiwara, Spectroscopic Ellipsometry-Principles and Applications (Wiley, 2007).

${ }^{16}$ A. Crovetto, A. Cazzaniga, R. B. Ettlinger, J. Schou, and O. Hansen, Thin Solid Films 582, 203 (2015).

${ }^{17}$ A. Crovetto, R. Chen, R. B. Ettlinger, A. C. Cazzaniga, J. Schou, C. Persson, and O. Hansen, Sol. Energy Mater. Sol. Cells 154, 121 (2016).

${ }^{18}$ G. E. Jellison and F. A. Modine, Appl. Phys. Lett. 69, 371 (1996); e-print arXiv:1011.1669v3.

${ }^{19}$ H. Fujiwara, S. Fujimoto, M. Tamakoshi, M. Kato, H. Kadowaki, T. Miyadera, H. Tampo, M. Chikamatsu, and H. Shibata, Appl. Surf. Sci. 421, 276 (2017).

${ }^{20}$ R. A. Synowicki, Phys. Status Solidi C 5, 1085 (2008).

${ }^{21}$ J. N. Hilfiker, N. Singh, T. Tiwald, D. Convey, S. M. Smith, J. H. Baker, and H. G. Tompkins, Thin Solid Films 516, 7979 (2008).

${ }^{22}$ S. Siebentritt, G. Rey, A. Finger, D. Regesch, J. Sendler, T. P. Weiss, and T. Bertram, Sol. Energy Mater. Sol. Cells 158, 126 (2016).

${ }^{23}$ S.-Y. Li, C. Hägglund, Y. Ren, J. J. Scragg, J. K. Larsen, C. Frisk, K. Rudisch, S. Englund, and C. Platzer-Björkman, Sol. Energy Mater. Sol. Cells 149, 170 (2016).

${ }^{24}$ T. Gokmen, O. Gunawan, T. K. Todorov, and D. B. Mitzi, Appl. Phys. Lett. 103, 103506 (2013).

${ }^{25}$ Ö. Demirciolu, M. Mousel, A. Redinger, G. Rey, T. Weiss, S. Siebentritt, I. Riedel, and L. Gütay, J. Appl. Phys. 118, 185302 (2015).

${ }^{26}$ A. Klein, T. Löher, Y. Tomm, C. Pettenkofer, and W. Jaegermann, Appl. Phys. Lett. 70, 1299 (1997).

${ }^{27}$ A. Crovetto, C. Yan, B. Iandolo, F. Zhou, J. Stride, J. Schou, X. Hao, and O. Hansen, Appl. Phys. Lett. 109, 233904 (2016).

${ }^{28}$ R. J. Archer, J. Opt. Soc. Am. 52, 970 (1962).

${ }^{29}$ A. Crovetto, A. Cazzaniga, R. B. Ettlinger, J. Schou, and O. Hansen, Sol. Energy Mat. Sol. Cells (to be published). 